\title{
INFIUENCE OF HEAT DISPERSION ON STEADY CONVECTION IN ANISOTROPIC POROUS MEDIA
}

\author{
by \\ Peder A. Tyvand \\ Department of Mechanics \\ University of Oslo \\ Oslo, Norway
}

\section{ABSTRACT}

The influence of heat dispersion on steady, buoyancy-driven convection is investigated theoretically. The motion takes place in an anisotropic porous layer with a unform basic flow and being uniformly heated from below. The fluid-saturated layer is horizontally isotropic with respect to permeability and thermal diffusivity. It is of infinite horizontal extent and bounded by two horizontal, perfectly heat-conducting planes. Dispersion effects at small Péclet numbers on the amplitude of the motion and on the heat transport are studied analytically. 


\section{INTRODUCTION}

Buoyancy-driven convection in flud.d-saturated porous layers has many important applications. Prominent among these are insulation techniques, flows in soils, aquifers, oil reservoirs and snow layers (Combarnous \& Bories, 1975). This type of flow is primarily caused by vertical density gradients due to gradients in temperature or in solute concentration in the fluid.

Free convection currents can be maintained only if there are buoyancy "sources" or "sinks" in connection with the flow field (e.g. a wastewater outlet). In our theoretical problem the "buoyancy source" is the lower boundary plane, kept at a constant temperature warmer than the fluid above. The "buoyancy sink" is the upper plane, kept at a constant temperature colder than the fluid below. Such a model allows a steady convection current with a constant flux of heat through the layer.

Dispersion effects on steady, nonlinear convection in isotropic media has been studied analytically by Neischloss \& Dagan (1975) and numerically by Kvernvold \& Tyvand (1979a), who also examined the stability of the motion. In the present paper, first approximation analytical results for anisotropic media will be found.

The dispersion effect on the onset of convection in an isotropic porous layer with a uniform basic flow, has been analyzed by Rubin (1974) and Weber (1975). The same problem for anisotropic media was studied by Tyvand (1977), hereafter referred to as paper I. The theory in I is here extended into the nonlinear regime of small supercritical Rayleigh numbers. Nonlinear convection in anisotropic media with neglect of dispersion was studied by Kvernvold \& Tyvand (1979b). 


\section{GOVERNING EQUATIONS}

A homogeneous, fluid-saturated porous layer is considered. It is bounded by two infinite horizontal planes with a distance $h$. The temperature difference between the lower and the upper plane is $\Delta T$. The $\mathrm{xy}-\mathrm{plane}$ is placed at the lower boundary plane, and the $\mathrm{z}$-axis is directed opposite gravity. The unit vectors $(\vec{i}, \vec{j}, \vec{k})$ correspond to $(x, y, z)$-directions. A uniform horizontal basic flow is assumed, defining positive $\mathrm{x}$-direction. The saturated porous medium is assumed to have permeabilities $\mathrm{K}_{\mathrm{H}}$ (horizontal) and $\mathrm{K}_{\mathrm{V}}$ (vertical), and thermal diffusivities $\kappa_{\mathrm{mH}}$ (horizontal) and $\kappa_{\mathrm{mV}}$ (vertical).

Dimensionless quantities are introduced by taking

$$
h_{1}\left(c_{p} \rho\right)_{m} h^{2} / \lambda_{m V}, \quad K_{m V} / h, \Delta T, \rho_{0} \nu K_{m V} / K_{V}
$$

as units of dimensionless length, time $t$, velocity $\vec{v}=(u, v, w)$, temperature $T$ and pressure $p$. The Darcy-Boussinesq equations may be written (I) :

$$
\begin{aligned}
& \vec{v}+\mathscr{U} \cdot(\nabla p-R T \vec{k})=0 \\
& \nabla \cdot \vec{v}=0 \\
& \left.\frac{\partial T}{\partial t}+\vec{v} \cdot \nabla T=\nabla \cdot(\mathscr{g}) \cdot \nabla T\right)
\end{aligned}
$$

The Rayleigh number is defined as

$$
\mathrm{R}=\mathrm{K}_{\mathrm{V}} \mathrm{ga} \Delta \mathrm{Th} / \nu \mathrm{K}_{\mathrm{mV}}
$$

In the equations above, $c_{p}=$ specific heat at constant pressure, $\rho$ = density, $\rho_{0}=$ standard density, $\lambda_{m V}=$ effective vertical thermal conductivity, $\nu$ = kinematic viscosity, $g$ = gravitational acceleration, $\alpha=$ coefficient of volume expansion. The subscript 
$m$ refers to the mixture of solid and fluid.

The dimensionless permeability tensor will be written

$$
Y=\xi(\vec{i} \vec{i}+\vec{j} \vec{j})+\overrightarrow{k k}
$$

where $\xi=\mathrm{K}_{\mathrm{H}} / \mathrm{K}_{\mathrm{V}}$. Introducing $n=\mathrm{k}_{\mathrm{mH}} / \mathrm{k}_{\mathrm{mV}}$, we may write the part of the dispersion tensor $\mathscr{D}$ which is due to molecular diffusion as

$$
n(\overrightarrow{i i}+\overrightarrow{j j})+\overrightarrow{k k}
$$

The relevant dimensionless number in connection with hydrodynamic dispersion is the Péclet number

$$
P=V d / k_{f}
$$

where $V$ is a characteristic, dimensional fluid velocity, $d$ a characteristic pore length, taken equal to the average grain diameter, and $k_{f}$ is the thermal diffusivity of the fluid.

The analysis will here be restricted to small Péclet numbers

$$
P \ll 1
$$

Poreh (1965) has derived the relevant tensor form

$$
\begin{aligned}
D / k_{f} & =\left[\beta_{1}+\beta_{2}\left[|\vec{v}|^{2} d^{2} / k_{f}{ }^{2}\right] \dot{\xi}+\beta_{3} d^{2}\left(\vec{v} \vec{v} / k_{f}{ }^{2}\right)\right. \\
& +\left[\beta_{4}+\beta_{5}\left(|\vec{v}|^{2} d^{2} / k_{f}^{2}\right)\right] \overrightarrow{k k}+\beta_{6} d^{2}|\vec{v}|(\overrightarrow{v k}+\overrightarrow{k v}) / \kappa_{f}{ }^{2}
\end{aligned}
$$

where

$$
\mathscr{E}=\vec{i} \vec{i}+\vec{j} \vec{j}+\overrightarrow{k k}
$$


Equation (10) is rewritten in a dimensionless form as

$$
\begin{aligned}
D=\eta(\vec{i} \vec{i}+\overrightarrow{j j}) & +\overrightarrow{k k}+\alpha_{1}|\vec{v}| 2 \mathscr{c}+\alpha_{2} \overrightarrow{v v}+\alpha_{3}|\vec{v}| 2 \overrightarrow{k k} \\
& +\alpha_{4}|\vec{v}|(\overrightarrow{v k}+\overrightarrow{k v})
\end{aligned}
$$

where the new dispersion coefficients $\alpha_{i}(i=1,2,3,4)$ are defined as

$$
\begin{array}{ll}
\alpha_{1}=\beta_{2} \frac{d^{2}}{h^{2}} \frac{k_{m V}}{k_{f}}, & \alpha_{2}=\beta_{3} \frac{d^{2}}{h^{2}} \frac{\kappa_{m V}}{k_{f}} \\
\alpha_{3}=\beta_{5} \frac{d^{2}}{h^{2}} \frac{k_{m V}}{k_{f}} & \alpha_{4}=\beta_{6} \frac{d^{2}}{h^{2}} \frac{k_{m V}}{k_{f}}
\end{array}
$$

Let $\omega$ denote the angle between $\vec{v}$ and the vertical unit vector $\vec{k}$. According to Poreh (1965) we may write

$$
\begin{aligned}
& \alpha_{i}=\sum_{j=0}^{\infty} \alpha_{1,2 j} \cos ^{2 j} \omega ; 1=1,2,3 \\
& \alpha_{4}=\sum_{j=0}^{\infty} \alpha_{4,2 j+1} \cos ^{2 j+1} \omega
\end{aligned}
$$

where the coefficients $\alpha_{m, n}$ are numbers depending solely on the geometry of the medium.

The dispersion coefficients $\gamma_{i}(1=1,2,3,4)$ applied in the linear theory (I) are given by

$$
\begin{aligned}
& \gamma_{1}=\alpha_{1,0}+\alpha_{2,0} \\
& \gamma_{2}=\alpha_{1,0} \\
& \gamma_{3}=\alpha_{1,0}+\alpha_{3,0} \\
& \gamma_{4}=\alpha_{4,1} \cos \omega
\end{aligned}
$$

Because mechanical dispersion must give positive contributions to the effective diffusion, $\gamma_{1}, \gamma_{2}$ and $\gamma_{3}$ are always positive. 


\section{STEADY NONLINEAR CONVECTION}

The basic state is given by

$$
\vec{v}_{S}=\overrightarrow{U i}_{i}, T_{S}=T_{0}-z, \quad p_{S}=p_{0}+R\left(T_{0} z-\frac{z^{2}}{2}\right)-U x / \xi
$$

$\mathrm{U}, \mathrm{T}_{0}$ and $\mathrm{p}_{0}$ are dimensionless constants.

The Pêclet number is

$$
P=U \frac{k_{m V}}{k_{f}} \frac{d}{h} \ll 1
$$

Arbitrary disturbances are added to the basic state :

$$
\left.\begin{array}{l}
\overrightarrow{\mathrm{v}}=\mathrm{U}_{\vec{i}}+\overrightarrow{\mathrm{v}}^{\prime}=\left(\mathrm{U}+\mathrm{u}^{\prime}\right) \vec{i}+\mathrm{v}^{\prime} \overrightarrow{\mathrm{j}}+\mathrm{w}^{\prime} \overrightarrow{\mathrm{k}} \\
\mathrm{T}=\mathrm{T}_{\mathrm{S}}+\theta^{\prime} \\
\mathrm{p}=\mathrm{p}_{\mathrm{S}}+\mathrm{p}^{\prime}
\end{array}\right\}
$$

The boundaries are taken to be impermeable and perfectly heat-conducting:

$$
w^{9}=\theta^{9}=0 \quad \text { at } z=0,1
$$

As in I a scalar function $\psi$ is introduced by the relation

$$
\overrightarrow{\mathrm{v}}^{\prime}=\nabla \times(\nabla \times \psi \overrightarrow{\mathrm{k}})=\left(\psi_{\mathrm{xz}}, \psi_{\mathrm{yz}},-\nabla_{1}{ }^{2} \psi\right)
$$

The temperature field is given by

$$
\theta^{\prime}=-\frac{1}{R}\left(\nabla_{1}^{2}+\frac{\hat{1}}{\xi} \frac{\partial^{2}}{\partial z^{2}}\right) \psi
$$

The boundary conditions may be written

$$
\psi=\psi_{z z}=0 \text { at } z=0,1
$$

The onset of convection is determined by a disturbance of the form

$$
\psi=A \sin \pi z \cdot \exp [i(k x+1 y)+\sigma t]
$$


with small amplitude $A$ so that the equations can be linearized. $k$ and $I$ are dimensionless wave numbers and $\sigma$ is the complex growth rate :

$$
\sigma=\sigma_{r}+1 \sigma_{1}
$$

Marginal stability, defined by $\sigma_{r}=0$, is associated with an oscillatory frequency

$$
\sigma_{i}=-k U\left[1+R \frac{\left(\alpha_{2}, 0+\alpha_{4,1}\right)\left(k^{2}+1^{2}\right)-2\left(\alpha_{1}, 0+\alpha_{3,0}\right) \pi^{3}}{k^{2}+1^{2}+\pi^{2} / \xi}\right]
$$

Accordingly, an $x$-dependent disturbance propagates with a phase velocity which may differ somewhat from $U$.

The Rayleigh number for the onset of convection is given by (I) :

$$
\begin{aligned}
R_{0} & =\left[1+\pi^{2} / \xi\left(k^{2}+I^{2}\right)\right]\left[\left(n+\alpha_{1}, U^{2}+\alpha_{2}, 0^{2}\right) k^{2}\right. \\
& \left.+\left(n+\alpha_{1,0} U^{2}\right) I^{2}+\left(1+\alpha_{1,0} U^{2}+\alpha_{3,0} U^{2}\right) \pi^{2}\right]
\end{aligned}
$$

The critical Rayleigh number is given by

$$
R_{C}=\pi^{2}\left\{\left[\left(n+\alpha_{1,0} U^{2}\right) / \xi\right]^{\frac{1}{2}}+\left[1+\left(\alpha_{1,0}+\alpha_{3,0}\right) U^{2}\right]^{\frac{1}{2}}\right\}^{2}
$$

Corresponding to the wave numbers

$$
k_{c}=0, \quad I_{c}=\pi\left[\frac{1+\left(\alpha_{1,0}+\alpha_{3,0}\right) U^{2}}{\xi\left(n+\alpha_{1,0} U^{2}\right)}\right]^{\frac{1}{4}}
$$

The results (27) - (28) depend crucially on the inequality

$$
\alpha_{2,0}>0
$$

To prove this inequality, consider for a moment a temperature field $T(x, y, t)$ being dispersed by a uniform flow $\vec{v}=\vec{U}$. The effective 
longitudinal diffusivity is $\mathrm{k}_{\mathrm{mH}}\left[1+\left(\alpha_{1,0}+\alpha_{2,0}\right) \mathrm{U}^{2}\right]$. The effective transverse diffusivity (in $\mathrm{y}$-direction) is $\mathrm{k}_{\mathrm{mH}}\left(1+\alpha_{1,0} \mathrm{U}^{2}\right)$. Anisotropy is irrelevant in this context. For isotropic media it is known from theory (Saffman 1960; Bear 1969) and experiments (Fried \& Combarnous 1971) that longitudinal dispersion is considerably larger than transverse dispersion. This implies that inequality (29) is valid.

According to the results from linear theory, we seek a steady finite amplitude solution consisting of rolls with axis aligned in the basic flow direction $\left(\frac{\partial}{\partial x}=0\right)$. The velocity is given by :

$$
\overrightarrow{\mathrm{v}}=\overrightarrow{\mathrm{i}}+\psi_{\mathrm{yz}} \vec{j}-\psi_{\mathrm{yy}} \overrightarrow{\mathrm{k}}
$$

The temperature field is given by

$$
T=T_{0}-z+\theta^{\prime}=T_{0}-z-\left(\psi_{y y}+\psi_{z z} / \xi\right) / R
$$

The dispersion tensor is written

$$
D=\mathscr{D}_{\mathrm{s}}+9
$$

where

$$
\text { פ }_{S}=\left[n+\left(\alpha_{1,0}+\alpha_{2,0}\right) U^{2}\right] \vec{i} \vec{i}+\left(n+\alpha_{1,0} U^{2}\right) \vec{j} \vec{j}+\left[1+\left(\alpha_{1,0}+\alpha_{3,0}\right) U^{2}\right] \vec{k} \vec{k}
$$

The nonlinear dispersion equation (4) may be written

$$
\left.-W^{\prime}+\vec{v}^{\prime} \cdot \nabla \theta^{\prime}=\nabla \cdot(D) S^{\circ} \cdot \nabla \theta^{\prime}\right)-\nabla \cdot(D \cdot \cdot \vec{k})+\nabla \cdot\left(D \cdot \cdot \nabla \theta^{\prime}\right)
$$

Principally this is an equation in $\psi$, if inserting from eqs.(12), (14) and (30-33) The solution, subject to the boundary conditions (22) is expressed as an amplitude expansion

$$
\psi=\psi^{(1)}+\psi^{(2)}+\ldots
$$

where $\psi^{(1)}$ is the first approximation, $\psi^{(1)}+\psi^{(2)}$ the second 
approximation and so on. The Rayleigh number excess above its value at the onset of convection is expanded in the same way

$$
R-R_{0}=R_{1}+R_{2}+\cdots
$$

Assume $v=0$ at $y=0 . \psi^{(n)}$ may then be written

$$
\psi^{(n)}=A_{n} \cos 1 y \sin \pi z+\sum_{p, q} B_{p q}^{(n)} \cos p 1 y \sin q \pi z
$$

where

$$
B_{p q}^{(n)}=B_{p q}^{(n)}\left(A_{1}, \cdots, A_{n-1}\right)
$$

The system will be solved to second order in the amplitude expansion. The results will be valid at slightly supercritical Raylelgh number only. The increase in the Péclet number due to the convection current will then hardy violate inequality (17).

In order to solve the system to second order, the solvability condition of the third order equation must be applied. We assume that the basic flow velocity is fixed, i.e. of zeroth order in the amplitude. Then just the following six dispersion coefficients will enter our calculations

$$
\alpha_{1,0}, \alpha_{1,2}, \alpha_{2,0}, \alpha_{3,0}, \alpha_{3,2}, \alpha_{4,1}
$$

From eqs.(12) and (14) the dispersion tensor with just six coefficients is

$$
\begin{aligned}
\mathscr{Q} & =\eta\left(\overrightarrow{i \vec{i}+\vec{j} j)}+\left(\alpha_{1,0}|\vec{v}|^{2}+\alpha_{1,2} w^{2}\right) \xi+\alpha_{2,0} \vec{v} \vec{v}\right. \\
& +\left(1+\alpha_{3,0}|\vec{v}|^{2}\right) \overrightarrow{k k}+\alpha_{3,2} w^{2} \overrightarrow{k k}+\alpha_{4,1} w(\overrightarrow{v k}+\overrightarrow{k v})
\end{aligned}
$$


By subtracting 9$)_{\mathrm{s}}($ eq. 33$)$ we find

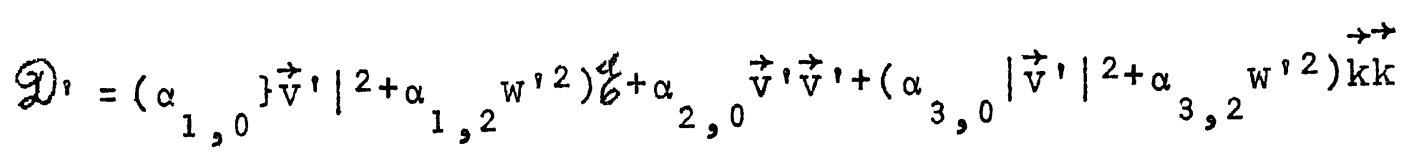

$$
\begin{aligned}
& +\alpha_{4,1} w^{\prime}\left(\vec{v} \cdot \vec{k}+\overrightarrow{k v} v^{\prime}\right)
\end{aligned}
$$

where we have neglected terms which cancel in eq. (34) when $\partial / \partial x=0$ By inserting eqs. (30), (31), (33) and (40) into (34), the governing equation for $\psi$ is found to be :

$$
\begin{aligned}
& {\left[\left(n+\alpha_{1,0} U^{2}\right) \frac{\partial^{2}}{\partial y^{2}}+\left(1+\left(\alpha_{1,0}+\alpha_{3,0}\right) U^{2}\right) \frac{\partial^{2}}{\partial z^{2}}\right]\left(\psi_{y y}+\psi_{z z} / \xi\right)+R \psi_{y y}} \\
& =\left(\psi_{\mathrm{yz}} \frac{\partial}{\partial \mathrm{y}}-\psi_{\mathrm{yy}} \frac{\partial}{\partial \mathrm{z}}\right)\left(\psi_{\mathrm{yy}}+\psi_{\mathrm{zz}} / \xi\right)-\mathrm{R}\left(\alpha_{1,0}+\alpha_{3,0}\right) \frac{\partial}{\partial \mathrm{z}} \psi_{\mathrm{yz}}{ }^{2}+\mathrm{R}\left(\alpha_{2,0}+\alpha_{4,1}\right) \psi_{\mathrm{yz}} \psi_{\mathrm{yyy}} \\
& -R\left(\alpha_{1,0}+\alpha_{1,2}+\frac{1}{2} \alpha_{2,0}+\alpha_{3,0}+\alpha_{3,2}+\frac{3}{2} \alpha_{4,1}\right) \frac{\partial}{\partial z} \psi_{y y^{2}} \\
& -\frac{\partial}{\partial y}\left[\left(\alpha_{1,0} \psi_{y z}{ }^{2}+\left(\alpha_{1,0}+\alpha_{1,2}\right) \psi_{y y}{ }^{2}\right) \frac{\partial}{\partial y}\left(\psi_{y y}+\psi_{z z} / \xi\right)\right] \\
& -\frac{\partial}{\partial z}\left[\left(\left(\alpha_{1,0}+\alpha_{3,0}\right) \psi_{y z}{ }^{2}+\left(\alpha_{1,0}+\alpha_{1,2}+\alpha_{3,0}+\alpha_{3,2}\right) \psi_{y y}{ }^{2}\right) \frac{\partial}{\partial z}\left(\psi_{y y}+\psi_{z z} / \xi\right)\right] \\
& -\alpha_{2,0}\left(\psi_{y z} \frac{\partial}{\partial y}-\psi_{y y} \frac{\partial}{\partial z}\right)\left[\left(\psi_{y z} \frac{\partial}{\partial y}-\psi_{y y} \frac{\partial}{\partial z}\right)\left(\psi_{y y}+\psi_{z z} / \xi\right)\right] \\
& +\alpha_{4,1}\left(\psi_{y z} \frac{\partial}{\partial y}-\psi_{y y} \frac{\partial}{\partial z}\right)\left[\psi_{y y} \frac{\partial}{\partial z}\left(\psi_{y y}+\psi_{z z} / \xi\right)\right] \\
& +\alpha_{4,1} \frac{\partial}{\partial z}\left[\left(\psi_{y y} \psi_{y z} \frac{\partial}{\partial y}-\psi_{y y}^{2} \frac{\partial}{\partial z}\right)\left(\psi_{y y}+\psi_{z z} / \xi\right)\right]
\end{aligned}
$$

The equations up to third order in the amplitude expansion are given in the appendix. 
It is known that the dispersion coefficients are of the order $(d / h)^{2}$ in the case of isotropy : The analytical results of Saffman (1960) Imply

$$
\left.\begin{array}{l}
\alpha_{1,0}=\frac{1}{40} \frac{k_{m}}{k_{f}}\left(\frac{d}{h}\right)^{2} \\
\alpha_{2,0}=\frac{5}{120} \frac{k_{m}}{k_{f}}\left(\frac{d}{h}\right)^{2}
\end{array}\right\}
$$

All other coefficients $\alpha_{p_{2} q}$ are zero for isotropic media. A change from isotropy to anisotropy must be continuous. It may be shown that dispersion in arbitrary directions are given by linear combinations of $\alpha_{p, q}$ (see Moranville et al. 1977). Therefore, all nonzero dispersion coefficients are of the order $(d / h)^{2}$ in anisotropic media as well.

From now on the following simplification is made: All products of two or more dispersion coefficients $\alpha_{p, q}$ are neglected. Terms of second order in $(d / h)$ are thus retained, while terms of fourth and higher order are neglected. This is consistent with the average continuum description of a porous medium (Bear 1972,p. 19) which requires that $d / h$ is small.

The solution of eq. (41) to second order in the amplitude is

$$
\left.\begin{array}{rl}
\psi & =\psi_{1}+\psi_{2}=A_{1} \cos 1 y \sin \pi z \\
& +{ }_{02}^{(2)} \sin 2 \pi z+B_{22}^{(2)} \cos 21 y \sin 2 \pi z
\end{array}\right\}
$$


To the order $(d / h)^{2}$ we find

$$
\begin{aligned}
& A_{1}=R_{2}^{\frac{1}{2}}\left[\frac{I^{2}}{8} \frac{I^{2}+\pi^{2} / \xi+R_{0}\left(\alpha_{1}, 0+\alpha_{1,2}+\alpha_{2,0}+\alpha_{3,0}+\alpha_{3,2}+2 \alpha_{4}, 1\right) I^{2}-R_{0}\left(\alpha_{1,0}+\alpha_{3,0}\right) \pi^{2}}{1+\left(\alpha_{1,0}+\alpha_{3,0}\right) U^{2}}\right) \\
& +\frac{3}{16}\left(1^{2}+\frac{\pi^{2}}{\xi}\right)\left(\alpha_{1,0}\left(1^{2}+\pi^{2}\right)^{2}+\alpha_{1,2} I^{4}+\left(\alpha_{1,2}+\frac{8}{3} \alpha_{2,0}+\alpha_{3,0}+\alpha_{3,2}+\frac{8}{3} \alpha_{4,1}\right)\right. \\
& \left.\left.I^{2} \pi^{2}+a_{3}, 0 \pi^{4}\right)\right]^{-\frac{1}{2}}
\end{aligned}
$$

$$
\begin{aligned}
& B_{02}^{(2)}=\frac{I^{2} \xi}{32 \pi^{2}} A_{1}^{2} \\
& \frac{I^{2}+\pi^{2} / \xi+R_{0}\left(\alpha_{1,0}+\alpha_{1,2}+\alpha_{2,0}+\alpha_{3,0}+\alpha_{3,2}+2 \alpha_{4,1}\right) I^{2}-R_{0}\left(\alpha_{1,0}+\alpha_{3}, 0\right) I^{2}}{1+\left(\alpha_{1,0}+\alpha_{3,0}\right) U^{2}} \\
& B_{22}^{(2)}=\frac{\pi^{3}}{24} A_{1}^{2}\left[\alpha_{1 ; 0}+\alpha_{3,0}+\left(\alpha_{1,0}+\alpha_{1,2}+\alpha_{3,0}+\alpha_{3,2}+\alpha_{4,1}\right) I^{2} / \pi^{2}\right]
\end{aligned}
$$

From now on, the wave number will be given its critical value (eq. 28) written as :

$$
I_{c}=\pi(\xi n)^{-\frac{1}{4}}\left(1+\frac{\alpha_{1,0}\left(1-n^{-1}\right)+\alpha_{3,0}}{4} U^{2}\right)+O(\alpha / h)^{4}
$$

Then $R_{0}=R_{c}$.

The dimensionless heat transport is given by

$$
H=-\vec{k} \cdot \overline{(\mathscr{D}) \cdot \nabla T)_{z}=0}
$$

The over-bar denotes horizontal average. The basic heat transport due to conduction and dispersion by the uniform basic flow is given by

$$
H_{S}=\vec{k} \cdot \mathscr{D}_{S} \cdot \vec{k}=1+\left(\alpha_{1,0}+\alpha_{3,0}\right) U^{2}
$$

The heat transport is conveniently represented by the Nusselt number :

$$
N=H / H_{S}=\left[\overline{\left(1+\left(\alpha_{1}, 0+\alpha_{3}, 0\right) \psi_{y z}{ }^{2}\right)\left(1+\left(\psi_{y y}+\psi_{z z} / \xi\right) / R\right)}\right]_{z=0}
$$


valid to the order $(d / h)^{2}$.

From eqs. (43)-(44) we find the Nusselt number to second order in the amplitude expansion :

$$
\begin{aligned}
N & =1+2 \frac{R-R_{c} 8}{R_{c}}\left\{1-\pi^{2}\left[\left(3+\frac{3}{2}(\xi n)^{-\frac{1}{2}}-\frac{1}{2}(\xi n)^{\frac{1}{2}}-2 n\right) \alpha_{1,0}+\right.\right. \\
& +\frac{3}{2}\left((\xi n)^{-\frac{1}{2}}+1\right) \alpha_{1,2}+4 \alpha_{2,0}+\left(\frac{3}{2}-\frac{1}{2}(\xi n)^{\frac{1}{2}}-2 n\right) \alpha_{3,0} \\
& \left.\left.+\frac{3}{2} \alpha_{3,2}+4 \alpha_{4,1}\right]\right\}
\end{aligned}
$$

To this approximation the Nusselt number is not influenced by the basic flow. All we know about the dispersion coefficients (beside their order of magnitude $\left.(d / h)^{2}\right)$ is that $\alpha_{1,0}$ and $\alpha_{2,0}$ are always positive and $\alpha_{1,0}+\alpha_{3,0}>0$. In total, hydrodynamic dispersion may reduce or increase the Nusselt number. At present we are not able to find the appropriate conditions. For isotropic media, eq. (49) reduces to

$$
N=1+2 \frac{R-R_{c}}{R_{c}}\left[1-\pi^{2}\left(2 \alpha_{1}, 0+4 \alpha_{2}, 0\right)\right]
$$

which was first derived by Neischloss \& Dagan (1975). In the absence of dispersion, i.e. when $d / h \rightarrow 0$, eq. (49) reduces to

$$
N=1+2 \frac{R-R_{c}}{R_{c}}
$$

This is known from Kvernvold \& Tyvand (1979b, appendix). 


\section{SUMMARY AND CONCLUS IONS}

Steady finite amplitude convection in an anisotropic porous layer heated from below has been investigated. Hydrodynamic dispersion has been taken into account, at small Péclet numbers. An amplitude expansion has been applied to solve the nonlinear problem. The solution to second order in the amplitude expansion and in the ratio of grain diameter to layer depth has been found.

The Nusselt number to second order only gives the slopes at which the Nusselt number curves start out from $R=R_{c}$. Except when $\xi / n$ is very small, these curves start out with a strong curvature (see Kvernvold \& Tyvand 1979b, fig. 2). Thus the Nusselt number to second order is usually not a good approximation, but it contains interesting information: Dispersion yields an Influence

of anisotropy on the Nusselt number to second order. This anisotropy influence is implicit through the dispersion coefficients, and explicit through the factors by which $\alpha_{1,0}, \alpha_{1,2}$ and $\alpha_{3,0}$ are multiplied in eq. (49). The uniform basic flow does not influence the Nusselt number to second order.

Our results indicate that the combination of anisotropy and dispersion effects may have a profound influence on the heat transfer through a porous layer. This influence may be much stronger than the isolated effects of anisotropy and dispersion, studied by Kvernvold \& Tyvand (1979a, 1979b). This is due to the nonlinear character of the problem, by which superposition of effects is strongly violated. 


\section{REFERENCES}

Bear, J., 1969. Hydrodynamic dispersion. In: R de Wiest (Editor), Flow through porous media. Academic Press, New York, $530 \mathrm{pp}$.

Bear, J., 1972 Dynamics of fluids in porous media. American Elsevier, New York, 764 pp.

Combarnous, M.A. and Bories, S.A., 1975. Hydrothermal convection in saturated porous media. Adv. Hydrosci. 10: 231-307.

Fried, J.J. and Combarnous, M.A., 1971. Dispersion in porous media. Adv. Hydrosci. $\underline{7}$ : 169-282.

Kvernvold, O. and Tyvand, P.A., 1979a. Dispersion effects on thermal convection in porous media. University of Oslo, Preprint Series no. 4.

Kvernvold, O. and Tyvand, P.A., 1979b. Nonlinear thermal convection in anisotropic porous media. J. Fluid Mech., 90: 609-624.

Moranville, M.B., Kessler, D.P. and Greenkorn, R.A., 1977. Directional dispersion coefficients in anisotropic porous media. Ind.Eng. Chem. Fundam. 16: 327-332.

Neischloss, $H_{0}$ and Dagan, G., 1975. Convective currents in a porous layer heated from below: The influence of hydrodynamic dispersion. Phys. Fluids, 18: 757-761. 
Poreh, M., 1965. The dispersivity tensor in isotropic and axisymmetric mediums. J.Geophys.Res., 70: 3909-3913.

Rubin, H., 1974. Heat dispersion effect on thermal convection in a porous medium layer. J.Hydrol., 21: 173-185.

Saffman, P.G., 1960.Dispersion due to molecular diffusion and macroscopic mixing in flow through a network of capillaries. J.Fluid Mech., 7: 194-208.

Tyvand, P.A., 1977. Heat dispersion effect on thermal convection in anisotropic porous media. J.Hydrol., 34: 335-342.

Weber, J.E., 1975. Dispersion effect on buoyancy-driven convection in stratified flows through porous media. J. Hydrol., 25: 59-70. 


\section{APPENDIX}

To find the equations up to third order in the amplitude expansion of eq. (41), we need the following expansions (from eqs.(35-36))

$$
\left.\begin{array}{l}
\psi=\psi^{(1)}+\psi^{(2)}+\psi^{(3)} \\
R-R_{0}=R_{1}+R_{2}
\end{array}\right\}
$$

By equating terms of first, second and third order we get the equations (A1), (A2) and (A3) respectively :

$$
\begin{aligned}
& \left.\begin{array}{l}
L \psi(1) \equiv\left[\left(\eta+\alpha_{1}, 0 U^{2}\right) \frac{\partial^{2}}{\partial y^{2}}+\left(1+\left(\alpha_{1}, 0+\alpha_{3}, 0\right) U^{2}\right) \frac{\partial^{2}}{\partial z^{2}}\right] \\
\left(\psi_{\mathrm{yy}}^{(1)}+\psi_{\mathrm{zz}}^{(1)} / \xi\right)+R_{0} \psi_{\mathrm{yy}}^{(1)}=0
\end{array}\right\} \\
& L \psi^{(2)}=-R_{1} \psi_{\mathrm{yy}}^{(1)}+\left(\psi_{\mathrm{yz}}^{(1)} \frac{\partial}{\partial y}-\psi_{\mathrm{yy}}^{(1)} \frac{\partial}{\partial z}\right)\left(\psi_{\mathrm{yy}}^{(1)}+\psi_{\mathrm{zz}}^{(1)} / \xi\right) \\
& \left.-R_{0}\left(\alpha_{1}, 0+\alpha_{3}, 0\right) \frac{\partial}{\partial z} \psi_{y z}^{(1)^{2}}+R_{0}\left(\alpha_{2}, 0+\alpha_{4}, 1\right) \psi_{y z}^{(1)} \psi_{y y y}^{(1)}\right\} \\
& -R_{0}\left(\alpha_{1}, 0+\alpha_{1,2}+\frac{1}{2} \alpha_{2,0}+\alpha_{3,0}+\alpha_{3,2}+\frac{3}{2} \alpha_{4,1}\right) \frac{\partial}{\partial z} \psi_{y y}^{(1)^{2}}
\end{aligned}
$$




$$
\begin{aligned}
& L \psi(3)=-R_{1} \psi_{y y}^{(2)}-R_{2} \psi_{y y}^{(1)}+\left(\psi_{y z}^{(1)} \frac{\partial}{\partial y}-\psi_{y y}^{(1)} \frac{\partial}{\partial z}\right) \\
& \left(\psi_{\mathrm{yy}}^{(2)}+\psi_{\mathrm{zz}}^{(2)} / \xi\right)+\left(\psi_{\mathrm{yz}}^{(2)} \frac{\partial}{\partial y}-\psi_{\mathrm{yy}}^{(1)} \frac{\partial}{\partial z}\right)\left(\psi_{\mathrm{yy}}^{(1)}+\psi_{\mathrm{zz}}^{(1)} / \xi\right) \\
& -\frac{\partial}{\partial y}\left[\left(\alpha_{1,0} \psi_{y z}^{(1)^{2}}+\left(\alpha_{1}, 0+\alpha_{1}, 2\right) \psi_{y y}^{\left.(1)^{2}\right)} \frac{\partial}{\partial y}\left(\psi_{y y}^{(1)}+\psi_{z z}^{(1) / \xi)}\right]\right.\right. \\
& -\frac{\partial}{\partial z}\left[\left(\left(\alpha_{1,0}+\alpha_{3,0}\right) \psi_{\mathrm{yz}}^{(1)^{2}}+\left(\alpha_{1,0}+\alpha_{1,2}+\alpha_{3,0}+\alpha_{3,2}\right)\right.\right.
\end{aligned}
$$

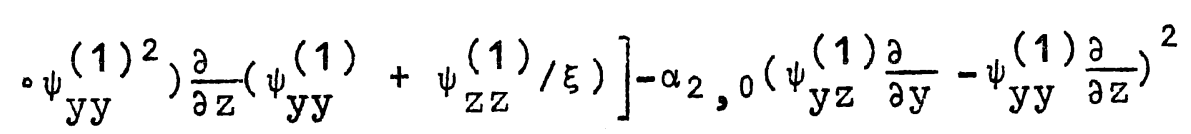

$$
\begin{aligned}
& \cdot\left(\psi_{\mathrm{yy}}^{(1)}+\psi_{\mathrm{zz}}^{(1)} / \xi\right)+\alpha_{4, I}\left(\psi_{\mathrm{yz}}^{(1)} \frac{\partial}{\partial y}-\psi_{\mathrm{yy}}^{(1)} \frac{\partial}{\partial z}\right)\left[\psi_{\mathrm{yy}}^{(1)} \frac{\partial}{\partial z}\left(\psi_{\mathrm{yy}}^{\left.(1)+\psi_{\mathrm{zz}}^{(1)} / \xi\right)}\right]\right. \\
& +\alpha_{4,1} \frac{\partial}{\partial z}\left[\left(\psi_{\mathrm{yy}}^{(1)} \psi_{\mathrm{yz}}^{(1)} \frac{\partial}{\partial y}-\psi_{\mathrm{yy}}^{(1)^{2}} \frac{\partial}{\partial z}\right)\left(\psi_{\mathrm{yy}}^{(1)}+\psi_{\mathrm{zz}}^{(1) / \xi)}\right]+0(\mathrm{~d} / \mathrm{h})^{4}\right]
\end{aligned}
$$

The solvability condition of the inhomogeneous equation (A2) determines $R_{1}=0$, accordingly $R_{2}=R-R_{0}$ to our approximation. The first order amplitude $A_{1}$ is determined from the solvability condition of (A3). 